\title{
Características estructurales de la web y su incidencia en la usabilidad. Análisis del caso en los portales municipales de la Comunidad de Madrid.
}

\section{Joaquín López del Ramo}

Facultad de Ciencias de la Comunicación, Universidad Rey Juan Carlos

\section{Resumen}

Este trabajo analiza las características estructurales de la web y su incidencia en la usabilidad, a través del estudio comparativo de los portales oficiales de los 17 ayuntamientos de la Comunidad de Madrid mayores de 50.000 habitantes, realizado durante el año 2008. Los objetivos fundamentales son: evaluar empíricamente cómo se realiza el tratamiento de estos aspectos en relación a los estándares ideales, determinar comparativamente las posibles tendencias y proponer un modelo coherente $\mathrm{y}$ extrapolable para la realización de otros trabajos similares. Los resultados revelan que en la mayor parte de los portales poseen una estructura jerárquica, mientras que el empleo de subwebs es relativamente significativo; predominan los criterios de organización del contenido centrados en el usuario; los sitios cuentan en su mayoría con mapa web que refleja la estructura del sitio y todos ellos poseen una URL significativa. Se infieren como principales conclusiones: a) el tratamiento de los factores estructurales de usabilidad presenta un nivel global superior al 70\% respecto a los valores ideales; b) la principal carencia se da en el número excesivo de subwebs, que resta consistencia los portales; c) predomina la variedad de la información sobre la profundidad de la misma; d) los resultados por ayuntamientos son bastante homogéneos.

Palabras clave: Usabilidad, estructura de la web, organización del contenido, Webs municipales, Comunidad de Madrid.

\begin{abstract}
Analysis of the structural characteristics of the web and its impact in usability, through a comparative study done during the year 2008, of the official web portals of the 17 municipalities with more than 50.000 inhabitants in the "Comunidad de Madrid". The main objectives are: to empirically evaluate this aspects in comparison with the ideal standards; to establish, through comparison, possible tendencies; and to propose a coherent and extrapolable model for similar works. The results show that most web portals have hierarchic structure and that the use of sub-webs is quite significant. Most of the content organization criteria are user centered, a majority have a web map that reflects the site structure and all of them use a significant URL. We infer, as main conclusions: a) the treatment of the structural usability factors shows a global level above $70 \%$ of the ideal values; b) the main error in usability is the excessive number of subwebs that play down the consistency of the web portals; c) the information variety predominates over the depth of the information; d) the results between city councils are very homogeneous.
\end{abstract}

Keywords: Usability, web structure, content organization, city council web sites, Community of Madrid. 


\section{Introducción}

La definición que J.J. Garrett (2002, p.11) da sobre la arquitectura de la información como "el diseño estructural del espacio de información para facilitar el acceso intuitivo al contenido", alberga explícitamente la idea de que las características estructurales tienen una incidencia primaria, directa y esencial sobre la facilidad de uso de la web. La arquitectura es el soporte, primero conceptual y luego funcional, a través del cual se organiza, canaliza y puede discurrir el flujo informativo de una web. El propio Garrett (2001) pone en paralelo la arquitectura con lo que él denomina Diseño de la Interacción, a través del cual se establece "cómo el usario interactúa con la funcionalidad del sitio".

En la misma línea, Steve Toub (2000, p.2) considera la Arquitectura de la Información como "el arte y la ciencia de organizar espacios de información con el fin de ayudar a los usuarios a satisfacer sus necesidades de información. La actividad de organizar comporta la estructuración, clasificación y rotulado de los contenidos del sitio web”. La visión de Veen (2001, p.64) es similar, cuando señala que "Los arquitectos de la información son esencialmente organizadores ("matchmakers"). Su trabajo consiste en entender íntimamente tanto el contenido de un sitio como las metas de la audiencia, y luego encontrar las concexiones entre ambos”.

Rossenfeld y Morville (2000, p.12) ahondan en esta idea y glosan las ventajas que tiene para el usuario una arquitectura de la información bien planeada, afirmando que "al tener acceso a un sitio por primera vez, los consumidores pueden entenderlo de inmediato y sin esfuerzos. Pueden encontrar pronto la información que requieren, con lo que se disminuye el tiepo (y el costo) que implica tanto encontrar la información como no encontrarla”. De nuevo, nos hayamos en la base de la usabilidad, y es Nielsen (2001, p.198), padre de la disciplina, quien sentencia esta realidad de forma categórica, cuando señala "Si la estructura es un desastre, no habrá diseño que la repare. Un arquitectura deficiente de la información siempre llevará a una deficiente usabilidad”. Nielsen y Loranger (2007, p.173) concluyen, en el polo opuesto, que "un sitio bien estructurado ofrece a los usuarios lo que quieren cuando lo quieren"

Ciertamente, las relaciones entre arquitectura y navegación son estrechísimas, por cuanto, siguiendo a Hassan, Martín e Iazza (2004) "La estructura del sitio web se refiere precisamente a las conexiones y relaciones entre páginas, a la topología de la red de páginas, así como a la granularidad de los elementos de información contenidos en las páginas; y la navegación a las posibilidades y forma en que cada página presenta las opciones de desplazamiento hacia otras páginas”. No obstante, y a pesar de su estrecha imbricación, los rasgos estructurales de la web pueden ser analizados por separado de la navegación, de la cual son condicionantes. La estructura "explica” en gran medida la navegación; aquella antecede a ésta, pero no a la inversa. Siguiendo de nuevo a Garrett (2001), simplemente se trata de niveles distintos, aunque posean relaciones subyacentes.

A la luz de lo señalado, la arquitectura de la información es un factor de importancia capital en la web, lo cual justifica su examen independiente de otros factores. La arquitectura puede considerarse como una categoría para cuyo análisis debieran tomarse en consideración un conjunto de indicadores que, primero, van a mostrar cómo está organizada la información y, en segundo término, el sentido de su empleo deja ver si los criterios seguidos corresponden a una buena o mala praxis de usabilidad. Esa es, precisamente, la orientación sobre la que pivota este artículo. 
Junto a lo anterior, cabe plantearse si es pertinente incorporar al análisis algunos otros elementos que no atañen a la arquitectura. Si la estructura es básicamente organización y canalización del contenido para, finalmente, facilitar la recuperación del mismo, quizás habría que pensar en que la "etiqueta” genérica del sitio, su URL también posee un valor estructural como elemento de recuperación, aunque a un nivel más genérico. De nuevo asumimos la referencia de Nielsen (2001, p.248) cuando sostiene que "nuestros estudios de usabilidad muestran que los usuarios leen un URL cuando tratan de descifrar la estructura de un sitio o los resultados potenciales del seguimiento de un hipervínculo". Probablemente, unos y otros estamos aludiendo a ese concepto más abierto, que Peter Morville (2004) denomina “encontrabilidad”.

Si desde el punto de vista teórico-conceptual, el corpus bibliográfico sobre usabilidad y estructura web puede considerarse amplio y creciente, el número de investigaciones dedicadas al desarrollo de modelos de análisis y de aplicaciones prácticas de los mismos se presenta notablemente más reducido; más aún si la visión se circunscribe al ámbito académico de las Ciencias de Comunicación. Entre los mismos puede citarse por su interés y cercanía a nuestros postulados el trabajo Arquitectura de la información y usabilidad en los web de la administración local catalana, de Zapata y Jiménez (2004), que analiza de manera comparativa 35 webs de ayuntamientos catalanes pertenecientes a poblaciones de más de 30.000 habitantes, evaluando aspectos tales como estructura de la información, etiquetado, navegación, diseño de interfaz o empleo de formularios. Asimismo, cabe descatacar el artículo Evaluación de la usabilidad en sistemas de información web municipales: metodología de análisis y desarrollo, realizado por Marcos y Rovira (2005) dentro del proyecto denominado Web semántica y sistemas de información documental.

El presente trabajo ha sido desarrollado en el marco del proyecto de investigación titulado "Contenidos, arquitectura y participación ciudadana en las web de los ayuntamientos de la Comunidad de Madrid mayores de 50.000 habitantes", perteneciente Programa de Creación y Consolidación de Grupos de Investigación URJC-Comunidad de Madrid entre los años 2008 y 2009. Dicho proyecto ha llevado a cabo una evaluación exhaustiva respecto de las características estructurales, operativas, de diseño visual, diseño editorial y contenido de los sitios web de ayuntamientos de la Comunidad de Madrid con mayor volumen poblacional, excluyendo la capital.

La elección de este rango de municipios se realiza por dos factores esenciales:

a) El hecho de contar con un volumen de población lo bastante grande como para presuponer y justificar la existencia de un portal municipal con un volumen y diversidad de contenidos suficientemente densos.

b) El notable desarrollo de los portales de los organismos públicos en general, y municipales en particular, a raíz de la implantación de sucesivos planes nacionales y autonómicos para el fomento de la llamada e-administración, lo que hace que se trate de un interesante campo de estudio que, por la propia naturaleza de estos portales, posee una muy relevante incidencia ciudadana. 


\section{Objetivos}

Tomando como base las anteriores premisas, este trabajo pretende alcanzar los siguientes objetivos:

a) Proponer un catálogo de características estructurales que tengan incidencia en la facilidad y claridad de uso de la web.

b) Evaluar empíricamente cómo se realiza el tratamiento de estos aspectos en relación a los estándares ideales dentro de los portales oficiales de ayuntamientos de la Comunidad de Madrid objeto de estudio.

c) Determinar comparativamente las posibles tendencias o discordancias, tanto en el conjunto de los portales analizados como individualmente.

d) Desarrollar y testar un modelo de análisis integral de los factores estructurales de la web susceptible de ser extrapolable para la realización de otros trabajos similares

\section{Metodología}

Se aborda el estudio de los elementos estructurales de las webs de los ayuntamientos de la Comunidad de Madrid con una población igual o superior a los 50.000 habitantes, a excepción de la capital, según los datos del Instituto Nacional de Estadística a 1/1/2008. Esto supone 17 portales web de los siguientes municipios: Alcalá de Henares, Alcobendas, Alcorcón, Fuenlabrada, Getafe, Leganés, Móstoles, Torrejón de Ardoz, Collado-Villalba, Coslada, Majadahonda, Parla, Pozuelo de Alarcón, RivasVaciamadrid, Las Rozas, San Sebastián de los Reyes y Valdemoro. Los municipios más pequeños no se consideraron relevantes para los objetivos de la investigación por componer un conjunto excesivamente disperso y heterogéneo. Con la misma intención de salvaguardar la homogeneidad de la muestra, queda al margen del estudio la web del ayuntamiento de Madrid, dada su propia envergadura y escala, derivadas del especial status de esta ciudad.

Dentro del proyecto de investigación en el cual se inscribe este trabajo se procedió a un análisis heurístico dividido en tres fases: a) Definición del modelo de análisis, creación de la ficha de análisis y de la matriz de datos informática, tareas realizadas: entre los meses de marzo y junio de 2008; b) protocolo de procedimiento y vaciado de los datos, llevados a cabo entre junio y octubre de 2008; c) Análisis de los datos y obtención de los resultados: realizados entre noviembre de 2008 y enero de 2009. Para el estudio de las características estructurales de la web se define como unidad de análisis cada uno de los 17 portales que comprende la investigación. Se adopta la teoría de Garrett (2001) sobre la existencia de 5 "niveles" respecto al diseño y análisis de sitios web centrados en la experiencia del usuario, de los cuales, en relación al tema concreto que aborda este trabajo, interesa el llamado "Nivel 3: La estructura", que comprende lo que habitualmente se conoce como "arquitectura de la información”, cuya expresión gráfica es el mapa de sitio web compuesto en forma de diagrama.

Con base en los fundamentos teórico-conceptuales expuestos por Louis Rosselfed y Peter Morville (2002), considerados autores de referencia en el tema específico de este trabajo, la estructura de la web, así como en los trabajos previos de Jacob Nielsen (2000), Nielsen y Loranger (2007), el equipo de investigación del proyecto creó una 
matriz de análisis general, integrada por 190 indicadores y sus correspondiente valores, que a su vez se agrupan en varios niveles (López del Ramo y Prieto Dávila, 2009). El primero de estos niveles es el estructural (Blueprints), cuyos indicadores aparecen relacionados seguidamente en la Tabla I:

\begin{tabular}{|c|c|}
\hline Indicador & Valores \\
\hline 1.Tipo de estructura general & $\begin{array}{l}\text { O 1. Jerárquica } \\
\text { O 2. Lineal } \\
\text { 3. Lineal con jerarquía } \\
\text { 4. Red } \\
\text { 5. Marco } \\
\text { 6. Mixta }\end{array}$ \\
\hline $\begin{array}{l}\text { 2.Esquema general de organización del } \\
\text { sitio }\end{array}$ & $\begin{array}{l}\text { O 1. Exacto } \\
\text { O 2. Ambiguo }\end{array}$ \\
\hline $\begin{array}{l}\text { 2.1. Criterio de organización } \\
\text { exacto } \\
\text { (Subordinado a indicador } 2= \\
\text { valor } 1 \text { ) }\end{array}$ & $\begin{array}{l}\text { O 1. Alfabético } \\
\text { O 2. Cronológico } \\
\text { O 3. Geográfico }\end{array}$ \\
\hline $\begin{array}{l}\text { 2.2. Criterio de organización } \\
\text { ambiguo } \\
\text { (Subordinado a indicador } 2= \\
\text { valor 2) }\end{array}$ & $\begin{array}{l}\text { O 1. Temático } \\
\text { 2. Funcional } \\
\text { 3. Por públicos } \\
\text { 4. Por metáforas } \\
\text { 5. Híbrido }\end{array}$ \\
\hline 3. Predomina la anchura o la profundidad & $\begin{array}{l}\text { 1. Anchura } \\
\text { 2. Profundidad }\end{array}$ \\
\hline $\begin{array}{l}\text { 4. Criterio de organización corporativo, o } \\
\text { centrado en el usuario }\end{array}$ & $\begin{array}{l}\text { O 1. Corporativo } \\
\text { O 2. Usuario }\end{array}$ \\
\hline 5.Existencia de subwebs & $\begin{array}{l}\text { O Sí } \\
\text { O No }\end{array}$ \\
\hline 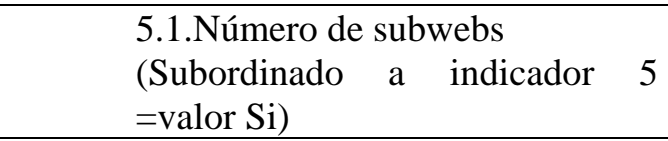 & Número \\
\hline $\begin{array}{l}\text { 5.2. Conexión entre las subwebs } \\
\text { y la estructura global } \\
\text { (Subordinado a indicador } 5 \\
=\text { valor } \mathrm{Si} \text { ) }\end{array}$ & $\begin{array}{l}\text { O Sí } \\
\text { O No }\end{array}$ \\
\hline 6. Existencia de Mapa Web & $\begin{array}{l}\text { O Sí } \\
\text { O No }\end{array}$ \\
\hline $\begin{array}{l}\text { 7- URL: } \\
\text { significativo, identificable } \mathrm{y} \text { fácil de } \\
\text { recordar }\end{array}$ & $\begin{array}{l}\text { O Sí } \\
\text { O No }\end{array}$ \\
\hline
\end{tabular}

Tabla I. Indicadores de la matriz de análisis sobre características estructurales de la web (Fuente: elaboración propia)

Con esta matriz se procedió a la codificación de todas las variables estructurales, a nivel del conjunto del sitio, en los 17 portales municipales objeto de estudio. Fueron aplicados criterios de codificación comunes, mediante la previa realización de pruebas de contraste y protocolos entre los miembros del panel de expertos encargados de este cometido. En paralelo a este proceso, se procedió a la creación de una base de datos en SPSS, cuyas variables y posibles valores correspondían a los de la matriz de análisis. Los datos primarios obtenidos tras el vaciado de los portales, se grabaron en dicha matriz informática para su posterior tratamiento. 
A fin de interpretar los datos de forma consistente y acorde con los objetivos de la investigación, se realizó un cuadro de equivalencias entre las variables de la matriz de análisis que actúan como indicadores y los valores idóneos que podrían obtenerse. De esta forma, quedó implícitamente diseñado un modelo que permitía evaluar comparativamente los resultados por contraste con un patrón de referencia, y así verificar el grado de aproximación de los resultados a los estándares idóneos de usabilidad respecto a las características estructurales de la web. En dicho cuadro aparecen relacionados todos los indicadores de la matriz de análisis con sus valores idóneos, y divididos en dos grupos: los cualitativos, cuyo valor sí incide en el grado de usabilidad del sitio, y los no cualitativos, que no poseen incidencia teórica respecto a la facilidad de uso. Los indicadores cualitativos aparecen relacionados en orden descendente de importancia por su mayor a menor peso sobre la usabilidad; en esta jerarquización, el autor aplica un criterio propio, que se tendrá en cuenta para ponderar los resultados de este trabajo.

\begin{tabular}{|l|l|l|}
\hline Indicador & Tipo de valor & Valor ideal \\
\hline 1. Tipo de estructura & & \\
\hline 2. Esquema general de organización & No cualitativo & indiferente \\
\hline 3. Predomino de anchura o de profundidad & No cualitativo & indiferente \\
\hline $\begin{array}{l}\text { 1. Criterio de organización corporativo o centrado } \\
\text { en el usuario }\end{array}$ & Cualitativo & $\begin{array}{l}\text { Centrado en el } \\
\text { usuario }\end{array}$ \\
\hline 2. Existencia de subwebs & Cualitativo & El menor \\
\hline $\begin{array}{l}\text { 2.1. Número de subwebs } \\
\text { 2.2. Conexión entre subwebs y estructura } \\
\text { general }\end{array}$ & Cualitativo & El menor \\
\hline 3. Existencia de Mapa web & Todas \\
\hline $\begin{array}{l}\text { 4. URL: nombre de dominio significativo, } \\
\text { identificable y fácil de recordar }\end{array}$ & Cualitativo & $\mathrm{Si}$ \\
\hline
\end{tabular}

Tabla II. Equivalencias entre indicadores de usabilidad a nivel estructural y sus valores idóneos. (Fuente: elaboración propia)

Con arreglo a los postulados de Lawrence Bardin (2002, p.28-29), en primer lugar se realiza un análisis categorial, orientado a clasificar y enumerar por frecuencia de presencia o ausencia los ítems de contenido significativos, que en este caso son los valores de los indicadores de la matriz considerados individualmente. Se trata de una exploración univariada, a partir de la cual se obtienen frecuencias, valores porcentuales $\mathrm{y}$ tendencias de los diferentes indicadores, tanto respecto al modelo ideal como a la media de todos los portales. El segundo término, se efectúa un análisis estructural (en el sentido empleado por Bardin), a fin de establecer relaciones de asociación o exclusión entre ítems de contenido, resultante, por tanto, de la lectura cruzada entre los indicadores estructurales considerados y los valores de los mismos en cada uno de los portales objeto de análisis.

\section{Resultados}

5.1. Análisis categorial: valores y frecuencias de los indicadores individuales

En este epígrafe se recogen los resultados obtenidos a partir de la lectura horizontal de los indicadores estructurales de la web, para observar sus valores y frecuencias 
individuales. En consonancia con lo expuesto en el apartado anterior, los indicadores se analizan separadamente, en dos grupos:

- No cualitativos: aquellos cuyo valor no implica un matiz positivo o negativo respecto a la usabilidad.

- Cualitativos: aquellos que dependiendo del valor que presenten sí inciden en la usabilidad.

\subsubsection{Indicadores no cualitativos (Tabla III)}

1. Tipo de estructura:

Los resultados obtenidos permiten constatar dos aspectos esenciales: a) Se observa que en la muestra analizada únicamente se utilizan dos tipos de organización estructural: jerárquica y mixta. b) Se aprecia un notable predominio de los portales con estructura jerárquica (14 de los 17 examinados, lo que equivale al 82,3\%), mientras que sólo en 3 se utiliza una estructura mixta $(17,7 \%)$.

2. Esquema de organización:

En el 100\% de los casos se utiliza un esquema de organización ambiguo.

\section{Predominio de la anchura/profundidad:}

La estructuración de contenidos presenta una disposición con predominio de la anchura en 15 de los 17 portales analizados, es decir, en el 88’2\%, mientras que la disposición en profundidad únicamente predomina en 2 de ellos, equivalentes al 11'8\%.

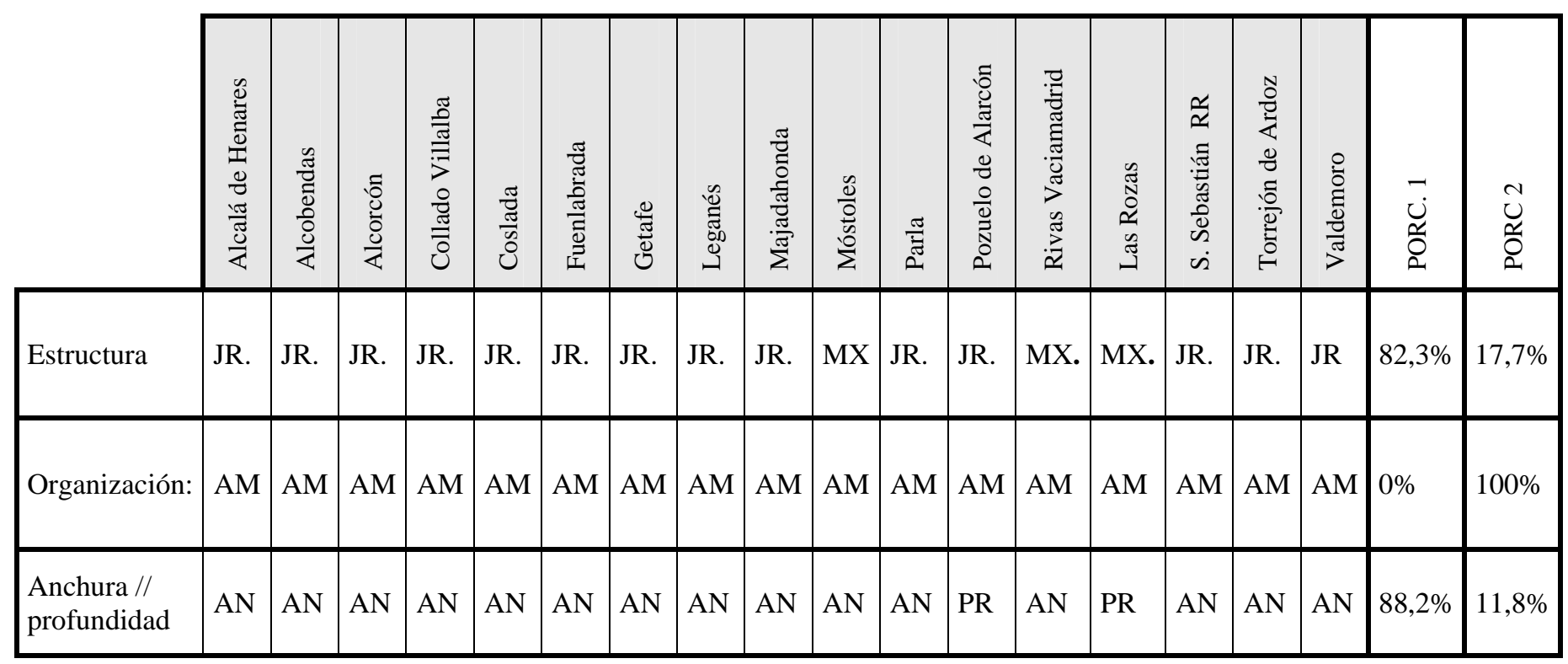

Tabla III. Resultados de los Indicadores no cualitativos: no inciden en el grado de usabilidad. Fuente: elaboración propia)

\subsubsection{Indicadores cualitativos (Tabla IV)}

1. Estructura organizada con criterio de usuario o corporativo:

Predominan con gran diferencia los portales en los que el criterio de estructuración del contenido refleja en punto de vista de los usuarios (13 de 17, equivalente al 76’5\%). Los 4 portales en los que se da el caso contrario (4 de 17) representan un porcentaje del 23’5. El modelo ideal responde al criterio de organización centrado en el usuario, siguiendo los postulados de Nielsen (2000, p.198), Rossenfeld y Morville (2002, p.12). 
2. Existencia y número de subwebs:

Teniendo en cuenta los criterios de codificación utilizados, en los que se adopta la consideración de subweb de Nielsen (2000, p.223) el empleo de este recurso se da en 9 de los 17 portales estudiados (52'9\%), en consecuencia, hay 8 portales, que no poseen ninguna subweb (47’1\%). Dentro de los portales que sí poseen subwebs, se observa una notable desigualdad en cuanto al número de las mismas. El sitio que más subwebs posee de todos los analizados cuenta con un total de $11 \mathrm{y}$, en orden decreciente, aparecen las siguientes cifras: uno con 10 , otro con 6 , dos con 3 , tres con 2 y un portal que sólo posee una subweb. El número medio de subwebs entre los portales analizados es de 4,4. Dado que el exceso de subsitios se considera una práctica poco deseable, por reducción el modelo ideal marca como más positiva la existencia del menor número posible. Este es el criterio más extendido entre los expertos y profesionales, por entenderse que el abuso de subsitios genera una fragmentación excesiva, falta de unidad estructural, visual y de navegación.

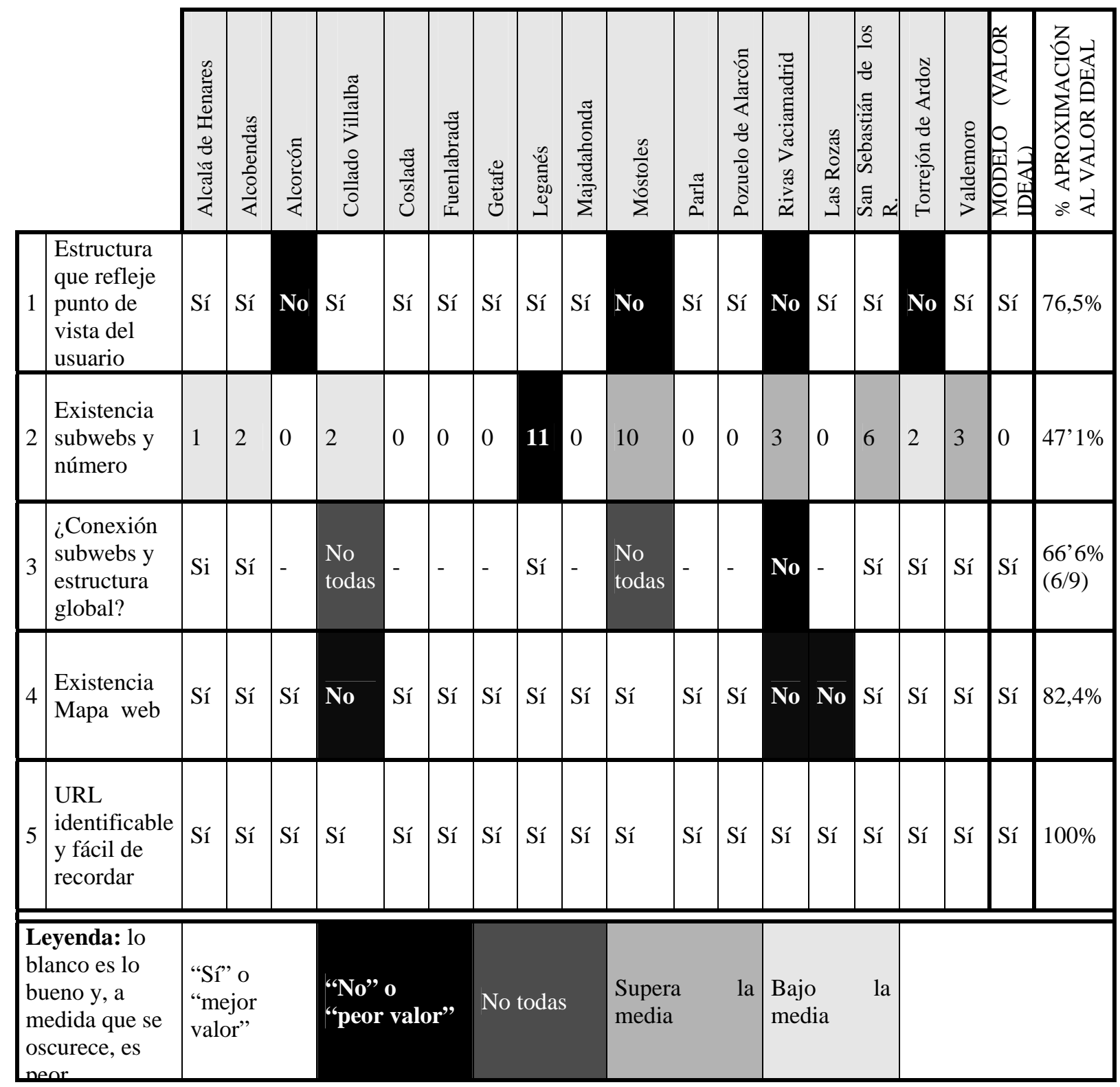

Tabla IV. Resultados de los Indicadores cualitativos: sí inciden en el grado de usabilidad. (Fuente: elaboración propia) 
3. Conexión de subwebs con estructura global:

Esta variable está subordinada a la anterior, ya que sólo es posible analizarla en aquellos portales que poseen subwebs. Se comprueba que 6 de los portales analizados cuentan con conexión entre todas las subwebs y su estructura general, que sería el valor ideal siguiendo lo expuesto por Rossenfeld y Morville (2002, p.56). En relación al total de sitios con subwebs (9), esto supone el 66'6\%. En orden descendente respecto al valor idóneo, la conexión entre subwebs y la estructura principal del sito sólo en algunos casos se da en 2 portales, que representan un valor porcentual respecto al total de los que tienen subwebs del 22'2\%. El ratio más bajo de este indicador, es decir, la ausencia completa de conexión entre subwebs y la estructura general del sitio, sólo se produce en un portal, lo que equivale al 11’1\% de los casos.

\section{Existencia de mapa web:}

Existe mapa web en 14 de los 17 portales analizados (82’3\%); únicamente carecen de este recurso estructural 3 de los portales examinados. El modelo ideal indica como conveniente la presencia de este recurso, siguiendo de nuevo a Rossenfeld y Morville (2002, p.67), Nielsen y Loranger (2007, p.72).

5. URL significativa e identificable:

Todos los portales analizados registran un valor afirmativo en esta variable, lo que implica el $100 \%$ en grado de cumplimiento respecto al modelo ideal, que está referenciado a lo expuesto por Nielsen y Loranger (2007, p.111) y el mismo Nielsen (2000, p.247)

6. Lectura comparativa de los indicadores cualitativos:

Según su grado de aproximación al modelo ideal, los indicadores cualitativos considerados en el análisis se sitúan, de más a menos favorable, en el siguiente orden:

- URL significativa e identificable: $100 \%$ de los casos.

- Existencia de mapa web: 82'4\% de los casos.

- Estructura que refleja el criterio del usuario: 76’5\% de los casos.

- Conexión entre subwebs y la estructura global: 66’6\% de los casos.

- Existencia y número de subwebs: 47’1\% de los casos.

Teniendo en cuenta la aplicación de un criterio de jerarquización cualitativa entre estos indicadores (Tabla II), se aprecia que los dos indicadores con menor importancia cualitativa poseen porcentajes más elevados de cumplimiento de los estándares ideales, si bien los resultados de los demás indicadores, los más importantes, presentan asimismo un valor de aproximación al modelo ideal muy elevado, y sólo el último de ellos (existencia y número de subwebs) registra un valor ligeramente inferior al 50\%.

\subsection{Análisis estructural: cruce de datos entre indicadores y portales}

En este epígrafe se lleva cabo una lectura cruzada de los resultados, en sentido verticalhorizontal, tomándose como hilo conductor las columnas de las Tablas III y IV, es decir, los sitios de cada uno de los ayuntamientos objeto de estudio, para observar sus valores en las intersecciones con las variables y las tendencias que presentan.

\subsubsection{Portales por indicadores no cualitativos (Tabla III):}

- Los sitios de los ayuntamientos de Alcalá, Alcobendas, Alcorcón, Collado Villalba, Coslada, Fuenlabrada, Getafe, Leganés, Majadahonda, Parla, San Sebastián de los Reyes, Torrejón y Valdemoro están configurados con estas características: 
- Estructura jerárquica

- Esquema de organización ambiguo

- Predominio de la anchura sobre la profundidad.

Esta combinación de valores es la tendencia mayoritaria.

- Los sitios de los ayuntamientos de Móstoles y Rivas poseen esta configuración:

- Estructura mixta

- Esquema de organización ambiguo

- Predominio de la anchura sobre la profundidad.

- El sitio de Pozuelo de Alarcón presenta estas características:

- Estructura jerárquica

- Esquema de organización ambiguo

- Predominio de la profundidad sobre la anchura.

- El sitio de Las Rozas posee estas características:

- Estructura mixta

- Esquema de organización ambiguo

- Predominio de la profundidad sobre la anchura.

5.2.2. Portales por indicadores cualitativos (Tabla IV):

- Los sitios que registran un valor óptimo en todos los indicadores cualitativos (ratio 5/5) son seis: Coslada, Fuenlabrada, Getafe, Majadahonda, Parla y Pozuelo, lo que equivale al 35 ’ $\%$ del total.

- Los portales cuyo valor es óptimo en todos los indicadores cualitativos, menos en uno (ratio 4/5) son siete (el 41’1\%). En la mayoría de los casos el indicador donde no registran el valor óptimo es el mismo: existencia y número de subwebs, el segundo cualitativamente más importante de los considerados. A tenor de ello se relacionan a continuación los sitios, en orden creciente al número de subwebs que poseen, indicando éste entre paréntesis: Alcalá (1), Alcobendas (2), Valdemoro (3), San Sebastián de los Reyes (6) y Leganés (11). Esta enumeración posee un carácter jerárquico, dado que se fijó el criterio de que cuanto mayor sea el número de subwebs menor es la eficacia. Llama la atención el excesivo número de subsitios del portal de Leganés, que excede con mucho la media general y los niveles recomendables.

Los otros dos sitios con este mismo ratio son Alcorcón y Las Rozas; el primero de ellos obtiene resultado negativo en el indicador estructura organizada con criterio de usuario, que en función de la ponderación jerárquica de los indicadores, es el más importante en términos de usabilidad. El sitio de La Rozas no concuerda con el valor ideal en el indicador existencia de mapa web, si bien éste es el cuarto según el criterio jerárquico utilizado.

- Con tres valores por encima del nivel óptimo (ratio 3/5) figura el sitio del ayuntamiento de Torrejón de Ardoz (5’8\%). Sus dos indicadores con valor por debajo del ideal son los más jerárquicamente más importantes: estructura organizada con criterio de usuario y existencia y número de subwebs, si bien en éste último la desviación negativa es poco acusada (2/0). 
- Los sitios de los ayuntamientos de Móstoles y Collado Villalba poseen unos niveles bastante inferiores al promedio general, pues su ratio es 2/5; ambos representan el 11.7 de la muestra. Móstoles no cumple con el estándar ideal en los indiciadores estructura organizada con criterio de usuario, existencia y número de subwebs y conexión entre subwebs y estructura global, que son, por dicho orden, los más importantes del estudio; además, tiene 10 subwebs, cifra muy por encima de la media. En el caso de Collado Villalba, no se llega al nivel ideal en los indicadores existencia, número de subwebs y conexión entre subwebs y estructura global y existencia de mapa web, que en conjunto poseen un nivel de importancia inferior al del portal anterior, por lo que pueden considerarse ligeramente más favorables.

- El ratio más bajo del análisis (1/5) corresponde al sitio del ayuntamiento de Rivas Vaciamadrid, cuyos resultados en los cuatro indicadores más importantes están por debajo del valor idóneo. Su representatividad en el total de la muestra es del 5’8\%.

\section{Conclusiones}

Los portales analizados presentan un alto grado de concordancia respecto al criterio de estructuración de sus contenidos. Los rasgos mayoritarios son: estructura jerárquica, criterio de organización ambiguo y que refleja el punto de vista del usuario.

El tratamiento de los factores estructurales es cualitativamente correcto en términos de usabilidad, pues presenta un valor global de aproximación al nivel idóneo superior al $70 \%$, si bien se observa una desigual tendencia a la dispersión del contenido por la existencia de excesivas subwebs.

Hay un notable predomino de la anchura sobre la profundidad en la arquitectura informativa, lo que implica que en los portales analizados se tiende a primar la diversidad de los contenidos, es decir, que en general hay unos contenidos muy variados pero presentan poco nivel de especialización.

Los resultados por ayuntamientos presentan un alto grado de homogeneidad, mayor en los indicadores estructurales no cualitativos que en aquellos que sí tienen incidencia sobre la usabilidad, si bien, incluso en éstos los resultados obtenidos presentan un nivel de concordancia elevado, que puede cifrarse en toro al 70\%.

Se considera que el modelo de análisis responde a los objetivos del estudio, es exhaustivo, por cuanto abarca las principales características estructurales que afectan a la usabilidad, y aplicable, por todo ello podría ser extrapolarse para el estudio de los mismos factores en otras investigaciones.

\section{Recomendaciones}

La realización de este trabajo y el modelo de análisis creado al efecto, que está concedido de forma abierta y, por tanto, es susceptible de ser ampliable, modificable y adaptable a otros ámbitos temáticos, temporales o espaciales, suscita en sus autores la inquietud de profundizar sobre el mismo objeto de estudio. Ello se hace aún más deseable teniendo en cuenta la naturaleza dinámica de este campo de investigación, los cambios tecnológicos a los que está sujeto, que a su vez estimulan el avance de sus postulados teórico-conceptuales y, porqué no, la escasez de investigaciones empíricas existente. 
Sería interesante la realización de trabajos de la misma naturaleza con una escala geográfica más amplia, incluso en esferas distintas dentro de las administraciones públicas y fuera de ellas, así como una puesta en común de investigaciones similares en proyectos de mayor escala, que permitieran enriquecer y actualizar los modelos de análisis y revelaran tendencias generales de mayor calado y relevancia.

\section{Referencias}

Bardin, Lawrence (2002). El análisis de contenido. Madrid, Akal, 2002.

Garrett, Jesse James. (2001) Los elementos de la experiencia de Usuario (diagrama original) <http://www.jjg.net/elements/> [Consulta: 05/06/2009].

Garrett, Jesse James. (2002) The Elements of User Experience: User-Centered Design for the Web. Berkeley, New Riders Publishing. 2002.

Hassan, Yussef; Martín Fernández, Franciso.J. e Iazza, Ghzala. (2004). Diseño Web Centrado en el Usuario: Usabilidad y Arquitectura de la Información [on line]. "Hipertext.net", núm. 2, mayo 2004. < http://www.hipertext.net/web/pag206.htm > [Consulta: 05/07/2009].

López del Ramo, Joaquín; Prieto Dávila, Pablo (2009). Modelo de análisis de diseño y usabilidad para sitios web. Registro General de la Propiedad Intelectual. Número de registro M-0022857/2009.

Lopez, Laura. Méndez, Eva María. Sordi, Angela.(2002). "Evaluación de la accesibilidiad y usabilidad de los sitios de las bibliotecas pública catalanas”. Revista de biblioteconomía i documentaciò, Mayo-agosto 2002, nº 31, p. 17-51.

<http://e-archivo.uc3m.es:8080/dspace/bitstream/10016/865/1/EMendez_Item.pdf>

[Consulta: 03/06/2009].

Marcos, M. Carmen; Rovira, Cristòfol. (2005).“Evaluacion de la usabilidad en sistemas de información web municipales”, Congreso ISKO-España ( $7^{\circ}: 2005$ : Barcelona) p. 415- 432. <http://bd.ub.es/isko2005/marcos.pdf> [Consulta: 25/05/2009].

Morville, Peter (2004) Diseño de experiencias del usuario (2004)

$<$ http://iainstitute.org/es/translations/000370.html> [Consulta: 12/06/2009].

Nielsen, Jacob; Loranger, Hoa (2007). Usabilidad. Prioridad en el diseño web. Madrid, Anaya Multimedia, 2007.

Nielsen, Jacob. Usabilidad. Diseño de sitios web (2000). Madrid, Pearson Educación, 2000.

Rosenfeld, Louis. y Morville, Peter(2002). Arquitectura de la información para el WWW. México, McGraw-Hill, 2002.

Toub. Steve (2000), Evaluating Information Architecture. A Practical Guide to Assessing Web Site Organization. Argus Center for Information Architecture. $<$ http://argus-acia.com/white_papers/evaluating_ia.pdf> [Consulta: 05/07/2009].

Veen, J. (2001) Arte y ciencia del diseño web. Madrid, Pearson Educación, 2001.

Zapata, Mónica; Jiménez, Eva. (2004) “Arquitectura de la informació i usabilitat als lloc web de l'administració local catalana”. Jornades Catalanes d'Informació i Documentació (Barcelona), 2004, pp. 161-182. <http://www.cobdc.org/jornades/9JCD/comunicacions.html $>$ [Consulta: 05/07/2009]. 